\title{
CHARACTERIZATION OF FLICKER NOISE IN GaN BASED MODFETS AT LOW
}

\section{DRAIN BIAS}

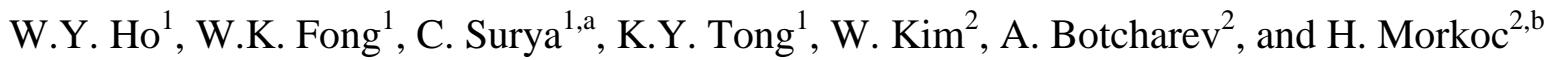 \\ ${ }^{1}$ Department of Electronic Engineering, The Hong Kong Polytechnic University, Hong Kong. \\ ${ }^{2}$ Department of Electrical and Computer Engineering and Coordinated Science Laboratory, \\ University of Illinois, U.S.A. \\ a email: ensurya@polyu.edu.hk \\ ${ }^{\mathrm{b}}$ Present address: Virginia Commonwealth University, Richmond, VA 23284.
}

\section{Cite this article as: MRS Internet J. Nitride Semicond. Res. 4S1, G6.5(1999)}

\begin{abstract}
We report systematic characterizations of flicker noise in GaN based MODFETs. Flicker noise was measured across the channel of the devices from room temperature to $130 \mathrm{~K}$. The voltage noise power spectra, $S_{V}(f)$ were found to be proportional to $1 / f^{\prime}$, where $\gamma$ depends on the device temperature as well as the gate bias. Study of $S_{V}(f)$ as a function of the biasing condition was conducted in detail and was found to vary as $V_{D}{ }^{2} /\left(V_{G^{-}} V_{T}\right)^{\beta}$ where $\beta$ changes with temperature from about 2.1 at room temperature to about 0.9 at $130 \mathrm{~K}$. Analyses of the data showed that the noise originated from thermal activation of carriers to localized states in the channel area. The data suggested that the trapping and detrapping of carriers did not lead to fluctuations in the carrier concentration as postulated in the McWhorter's model. However, more work is needed to determine if surface mobility fluctuations played key role in the $1 / f$ noise.
\end{abstract}

\section{INTRODUCTION}

In the past several years, an intensive effort in the development of III-V nitride based devices has been expended [1]. The high electron saturation velocity, large breakdown field, high thermal conductivity, and superb mechanical stability of GaN are among its properties that make it an excellent candidate for high-temperature and high-power applications at high frequencies [2]. The accomplishments made over the past couple years clearly demonstrate the rapid pace in which III-V nitride based FETs are being developed. However, the investigation of noise properties, particularly low-frequency noise has not yet gotten the necessary attention.

Flicker noise is an important figure-of-merit for semiconductor devices. It represents the lower limit of the signal that can be processed by the device. Previous studies of the phenomenon had shown that flicker noise in electron devices often arises from material defects which manifest themselves as the trapping and detrapping of carriers by localized states and grain boundary motion [3-5]. The objective of our work is to identify the underlying physical noise processes to pave the way for improving the noise properties of the device. To accomplish this, we studied the voltage noise power spectral density, $S_{V}(f)$, over a wide range of temperatures and biases to investigate the role of localized states in the fluctuation process.

\section{EXPERIMENT}

Gallium nitride based MODFETs were fabricated with reactive MBE on (0001) sapphire 
substrates. An $800 \AA \mathrm{AlN}$ buffer layer was first grown at $800^{\circ} \mathrm{C}$ followed by the deposition of a $1.2 \mu \mathrm{m}$ thick undoped GaN layer. The two-dimensional electron gas was formed at the heterointerface between the i-GaN layer and a $30 \AA \mathrm{i}-\mathrm{AlGaN}$ spacer layer which serves to separate the 2-dimensional electron gas from the ionized dopants to increase the mobility of the carriers. The electron donor layer consists of a $120 \AA$-thick $\mathrm{Si}$ doped $\mathrm{AlGaN}$ layer of concentration $5 \times 10^{18} \mathrm{~cm}^{-3}$. Ti/Al/Ti/Au $(200 \AA / 1700 \AA / 450 \AA / 550 \AA)$ multilayers were deposited to form ohmic contacts to the drain and source regions. The gate length and gate width are $2 \mu \mathrm{m}$ and $40 \mu \mathrm{m}$, respectively.

The low-frequency noise of the device was characterized from room temperature down to $130 \mathrm{~K}$, with the gate biased at a fixed voltage and the conduction channel was current biased with a passive current source. The detailed experimental set-up was described in previous publication [6].

\section{RESULTS AND DISCUSSIONS}

The experimental values for $S_{V}(f)$ at $500 \mathrm{~Hz}$ at $V_{G}=0.8 \mathrm{~V}$ and $V_{D}=0.2 \mathrm{~V}$ were found to be relatively insensitive to temperature change and were about $4 \times 10^{-12} \mathrm{~V}^{2} \mathrm{~Hz}^{-1}$ over the entire temperature range as shown in Fig. 1. However, $S_{V}(f)$ exhibited strong dependencies on both the gate and drain voltages.
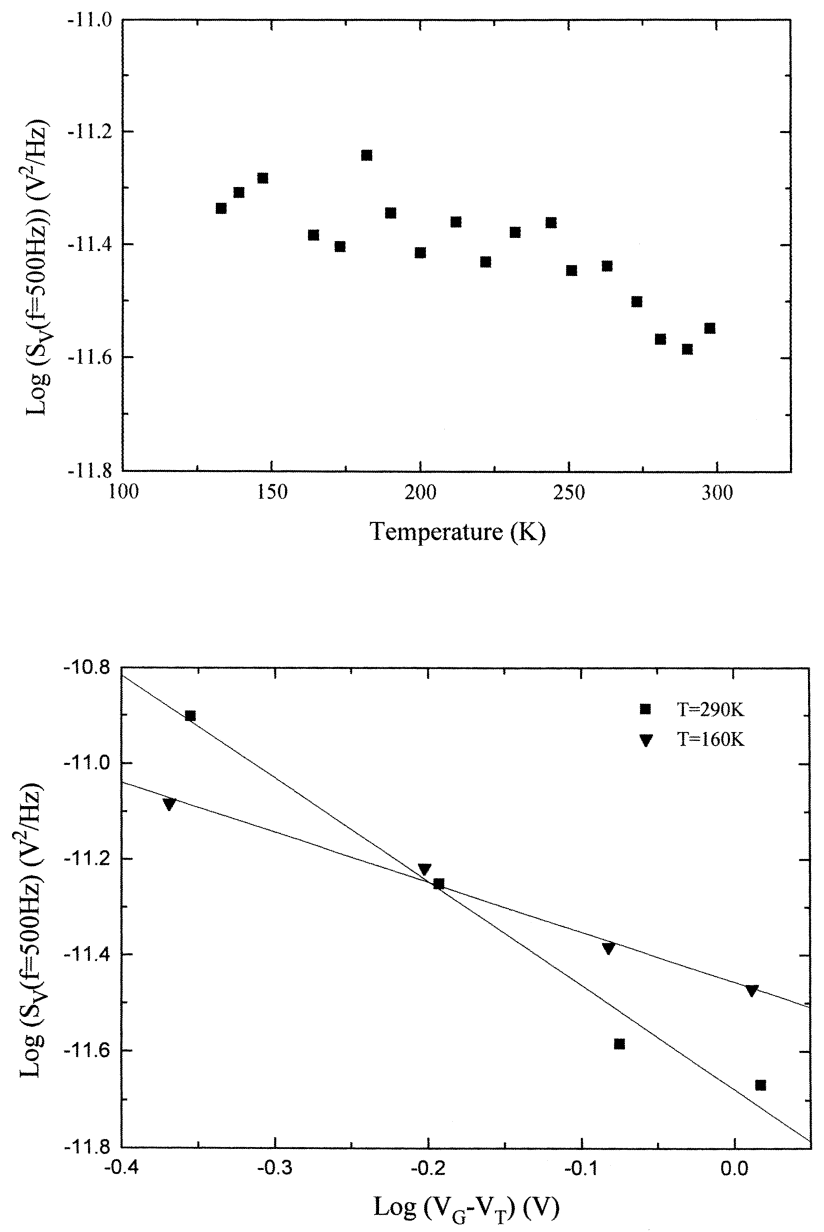

Fig. 1: The voltage noise power spectral density, $S_{V}(f=500 \mathrm{~Hz})$, over the conduction channel as a function of device temperature.
Fig. 2: $\log \left[S_{V}(f=500 \mathrm{~Hz})\right] \quad$ is plotted versus $\log \left(V_{G}-V_{T}\right)$ at $\mathrm{T}$ $=290 \mathrm{~K}$ and $\mathrm{T}=160 \mathrm{~K}$. 
The experimental results showed that $S_{V}(f) \propto V_{D}{ }^{2} /\left(V_{G}-V_{T}\right)^{\beta}$ where $\beta$ varied between 2.1 at room temperature to about 0.9 at $\mathrm{T}=130 \mathrm{~K}$. Typical results of $\log S_{V}(f)$ versus $\log \left(V_{G}-V_{T}\right)$ at $\mathrm{T}=290 \mathrm{~K}$ and $160 \mathrm{~K}$ are shown in Fig. 2, which clearly indicates changes in $\beta$. Typical plot of $\log S_{V}(f)$ versus $\log V_{D}$ at $263 \mathrm{~K}$ is shown in Fig. 3 for $f=500 \mathrm{~Hz}$. The slope of the best fit is 2.05, which demonstrates a quadratic relationship with $V_{D}$.

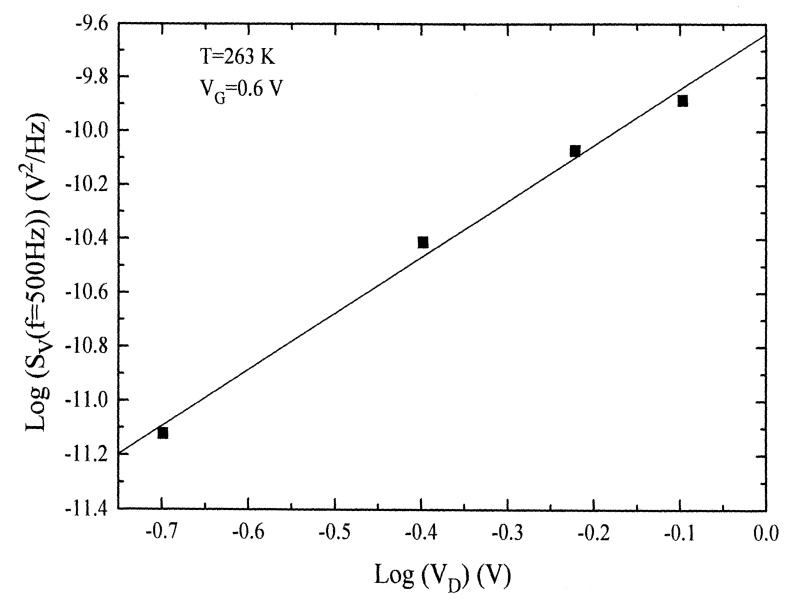

Fig. 3: $\log \left[S_{V}(f=500 \mathrm{~Hz})\right]$ is plotted versus $\log \left(V_{D}\right)$ at $V_{G}=$ $0.6 \mathrm{~V}$ and $\mathrm{T}=263 \mathrm{~K}$.

Flicker noise in semiconductor devices has been a subject of interest for many years $[6,7]$. Of the various models presented in the past, the thermal activation model proposed by Dutta and Horn [7] stands out in its success in explaining the temperature dependence of the noise. Application of the model in field-effect transistors was investigated by Surya and Hsiang [6] who suggested that $1 / f$ noise arises from thermal activation of carriers to traps at different energy levels. In this case the voltage noise power spectral density is

$$
S_{V}(f)=C \int d E \frac{N_{T}(E) \tau}{1+4 \pi^{2} f^{2} \tau^{2}}
$$

where $C$ is a proportionality constant and $E$ is the activation energy. It is shown that the Lorentzian peaks sharply at $E_{p}=-k_{B} \ln \left(2 \pi \tau_{0}\right)$. Consequently $1 / f$ noise is highly sensitive to trap distribution at $E_{p}$. It can be shown that $\gamma=1$ if $\left.N_{T}(E)\right|_{E \approx E p}$ is independent of $E ; \gamma>1$ if $\left.N_{T}(E)\right|_{E \approx E p}$ increases with $E$; and $\gamma<1$ if $\left.N_{T}(E)\right|_{E \approx E p}$ decreases with $E$. Since $E_{p}$ varies linearly with temperature, traps at energy level will be responsible for the observed noise as the device temperature is varied. Then $\gamma$ will vary according to the relationship as discussed above if $\frac{\partial N_{T}(E)}{\partial E}$ changes with energy.

The observation of a systematic variation of $\gamma$ as a function of $\mathrm{T}$ in our experimental data is indicative of a thermally activated process that underlies the noise. The experimental values of $\gamma$ versus $\mathrm{T}$ for $V_{G}=0.8 \mathrm{~V}$ and $V_{D}=0.2 \mathrm{~V}$ are indicated in Fig. 4 in which $\gamma$ decreased from 1.03 to about 0.85 when $\mathrm{T}$ varied from room temperature to $250 \mathrm{~K}$. Further decrease in the device temperature led to a gradual increase of $\gamma$ to 0.98 at $220 \mathrm{~K}$. Below 220K, $\gamma$ slowly decreased to about 0.9 as the device temperature was lowered to $130 \mathrm{~K}$.

Both traps at the GaAlN/GaN interface or along the line defects within the twodimensional electron gas are viable candidates for the observed $1 / f$ noise. Given the high 
concentration of line defects in $\mathrm{GaN}$, the latter mechanism is highly probable for our devices. To further investigate the physical origin of the noise we study the variations of $\gamma$ as a function of $\mathrm{V}_{\mathrm{G}}$. It is observed that $\gamma$ exhibited a systematic dependence in the applied gate bias. Typical results are shown in Fig. 5, for $\mathrm{V}_{\mathrm{D}}=0.2 \mathrm{~V}$ at $\mathrm{T}=250 \mathrm{~K}$ where $\gamma$ decreased initially from 0.9 to about 0.85 as $\mathrm{V}_{\mathrm{G}}$ varied from $0.4 \mathrm{~V}$ to $0.6 \mathrm{~V}$. Subsequent increase in $\mathrm{V}_{\mathrm{G}}$ led to increase in $\gamma$ to 0.96 at $\mathrm{V}_{\mathrm{G}}=1 \mathrm{~V}$. This can be explained by the change in band bending in the AlGaN layer with the application of a voltage bias resulting in a change in the trap energy levels with respect to the Fermi level in the device. Depending on the actual distribution of $N_{T}(E)$ it may lead to a change in $\gamma$. Base on these results it appears that the noise arises from trapping and detrapping at $\mathrm{GaN} / \mathrm{AlGaN}$ interface.
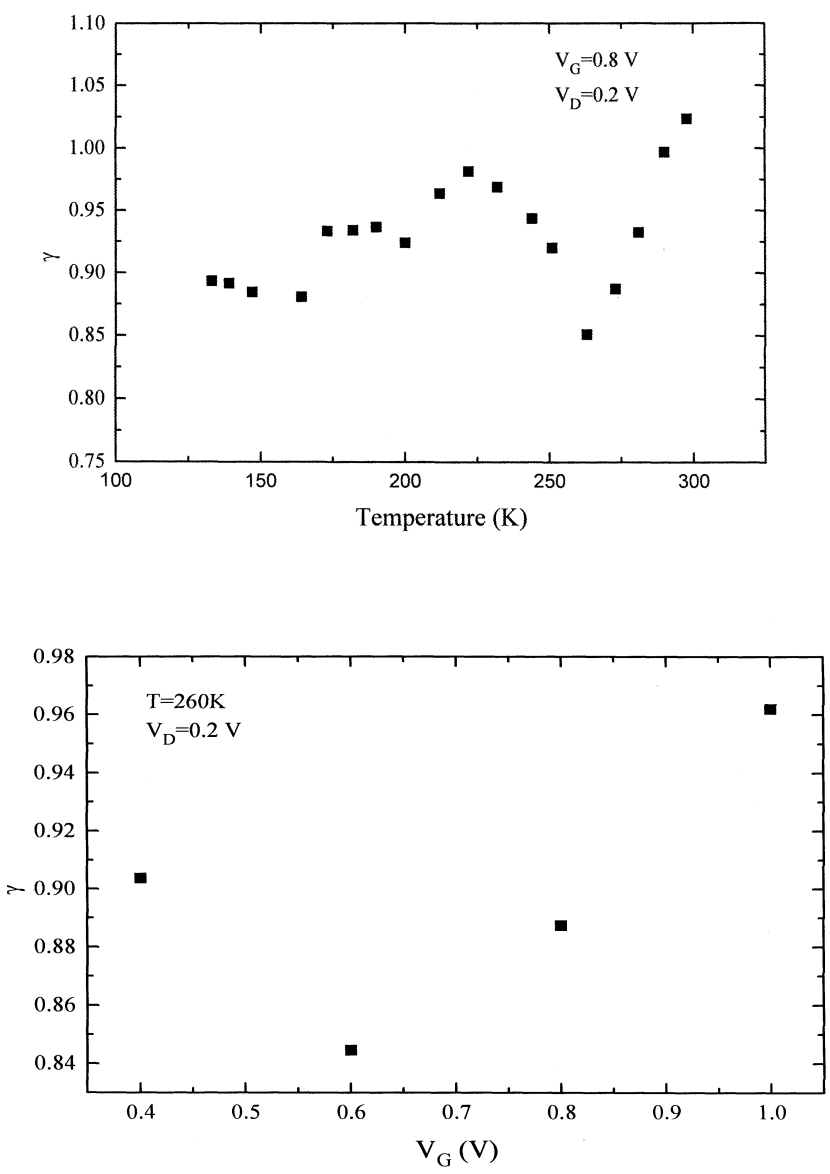

Fig. 4: The temperature dependence of the frequency exponent, $\gamma$.
Fig. 5: Gate bias dependence of the frequency exponent, $\gamma$, at $\mathrm{T}=$ $260 \mathrm{~K}$ and $V_{D}=0.2 \mathrm{~V}$.

McWhorter postulated that the capture of an electron by a trap causes the local modulation of the Fermi Level leading to fluctuations in the local carrier concentration. However, it was shown that the process may also lead to fluctuations in the carrier mobility due to Coulombic scattering. Studies on low-frequency noise in field-effect transistors showed that $S_{V}(f) \propto\left(V_{D} /\left(V_{G}-V_{T}\right)\right)^{2}$ for noise dominated by number fluctuations. On the other hand, for $1 / f^{\prime}$ noise dominated by surface mobility fluctuations, the dependence of $S_{V}(f)$ on $V_{G}$ arises from the screening effect by the two-dimensional electron gas which generally demonstrate a different $\mathrm{V}_{\mathrm{G}}$ dependence from number fluctuations and would exhibit stronger dependencies on temperature due to the variations of the screening parameter with $T$. The experimental results in Fig. 2 clearly deviated from the quadratic relationship as stipulated by numbers fluctuation model. However, a more definitive study that involves the characterization of random telegraph noise, 
which allows exact measurements of the fluctuations in the channel resistance resulting from single-electron events, is needed to conclusively prove the underlying fluctuating quantity of the noise. Such a phenomenon is typically seen in sub-micrometer size devices.

\section{CONCLUSION}

We have conducted systematic characterizations of $1 / \mathrm{f}^{\gamma}$ noise in GaN MODFETs. Detailed analyses of the magnitudes and $\gamma$ of the voltage noise power spectra showed that the noise originated from the thermal activation of carriers to localized states either at the $\mathrm{AlGaN} / \mathrm{GaN}$ heterointerface or line defects within the two-dimensional electron gas. Our experimental data suggest that trapping and detrapping at the heterointerface dominated the observed flicker noise. In addition, it was found that $S_{V}(f) \propto V_{D}^{2} /\left(V_{G}-V_{T}\right)^{\beta}$ where $\beta$ varied from about 2.1 at room temperature to about 0.9 at $130 \mathrm{~K}$. This suggests that the capture and emission of carriers by interface traps did not result in number fluctuations. A more detailed study of random telegraph noise is needed to confirm if surface mobility fluctuations due to Coulombic scattering played a major part in the observed noise.

\section{Acknowledgement}

The research conducted at the Hong Kong Polytechnic University is supported by an RGC

grant number POLYU 107/96E. The research at the University of Illinois was funded by grants from AFOSR and ONR under the program management of Drs. G.L. Witt, C.E.C. Wood, Y.S. Park, and M. Yoder.

\section{References}

[1] S.N. Mohammad and H. Morkoc, Progress in Quantum Electronics 20 (5-6), 361-525 (1996).

[2] H. Morkoc, S. Strite, G.B. Gao, M.E. Lin, B. Sverdlov, and M. Burns, J. Appl. Phys. 76 (3), 1363-1398 (1994).

[3] C. Surya, S.H. Ng, E.R. Brown, and P.A. Maki, IEEE Trans. Electron Devices 41 (11), 2016-2022 (1994).

[4] C. Surya, W. Wang, W.K. Fong, C.H. Chan, and P.T. Lai, Solid-State Electron. 39 (11), 1577-1580 (1996).

[5] W.Y. Ho and C. Surya, Solid-State Electron. 41 (9), 1247-1249 (1997).

[6] C.H. Cheng and C. Surya, Solid-State Electron. 36 (3), 475-479 (1993).

[7] P. Dutta and P.M. Horn, Rev. Modern Phys. 53 (3), 497-516 (1981). 